\title{
LETTER
}

\section{Evaluating mortality in an intensive care unit requires extended survival models}

\author{
Martin Wolkewitz ${ }^{*}$ and Harriet Sommer \\ See related research by Ju et al., http://ccforum.com/content/17/5/R230
}

In a recent issue of Critical Care, we read with great interest that admission at nighttime is associated with poor outcomes in intensive care units (ICUs) [1]. However, Ju and colleagues showed cumulative survival plots predicting a poor survival in the ICU of less than $20 \%$ after 2,000 ICU-hours for patients for both groups (nighttime or office time) despite the fact that 148 out of 175 patients (84.6\%) survived the ICU (nighttime group) and patients admitted during office time have even an ICU survival of 96.4\%. These large discrepancies are very confusing for the reader. We argue that this result is subject to a common type of survival bias [2], which we explain as follows. A fundamental assumption for calculating Kaplan-Meier survival curves is that censoring is non-informative in the sense that the hazard of death remains unchanged when a censoring event occurs. Presumably, the authors treated discharged patients as censored (displayed as crosses in their figures [1]). It is obvious that this assumption does not hold since discharged patients are usually in a better health condition than patients who stay in the ICU. This wrong assumption led to artificially reduced survival plots. The statistical solution for this is to treat discharge as a competing event for death in the ICU $[2,3]$ since the cumulative probability of death in the ICU depends not only on the ICU death hazard rate but also on the discharge rate.

Evaluating ICU mortality requires advanced statistical methods. Incorrect results may lead to wrong conclusions for clinicians and finally impact patient care. For that reason, it is crucial to have adequate and valid predictions for this important clinical outcome in hospital epidemiology.

\section{Authors' response}

\section{Min-Jie Ju, Jian-Feng Luo, and Zhe Luo}

We were delighted to have the opportunity to receive the advice of Wolkewitz and Sommer. Honestly, their criticism was constructive and helped us to consolidate the results of our study [1]. Hence, we might set a competing risks model to improve our analysis.

Survival analysis is a good tool when researchers take into account the time until an event occurs and the censored data. One of the common mistakes when applying survival analysis is not to include the censored data [4]. The primary outcome of our study was the ICU mortality, not the hospital mortality. The definition of 'event' was that patients died during their stay in the ICU. If the patients were alive after they were discharged from the ICU, they were censored (left-censored). We agree with Wolkewitz and Sommer that discharged patients might be in a better health condition. Therefore, we also applied survival

\footnotetext{
* Correspondence: wolke@imbi.uni-freiburg.de

Institute of Medical Biometry and Medical Informatics, Stefan-Meier-Str. 26, 79104 Freiburg, Germany
}

analysis in the propensity score matching cohort where the discharged ratio were balance distributed between nighttime and office hours (Table 1). Thus, the censoring bias was minimized and would not influence the primary result.

As for large discrepancies between the cumulative ICU survival after 2,000 ICU-hours and ICU survival probability, we think they were conceived differently. The former meant a property of any patient's survival time longer than

Table 1 Comparison of discharged ratio between nighttime and office hours admissions in 1:1 propensity score matching group

\begin{tabular}{|c|c|c|c|}
\hline & \multicolumn{2}{|c|}{ Intensive care unit outcome } & \multirow[t]{2}{*}{$P$ value } \\
\hline & Discharged (alive) & Dead & \\
\hline Office hours & 155 & 20 & 0.272 \\
\hline Nighttime & 148 & 27 & \\
\hline
\end{tabular}

${ }^{\mathrm{a} C h i-s q u a r e d}$ test. 
$t$ time, and the latter meant the ratio of the number of patients who were alive during ICU stay to the total number of patients. Accordingly, the analysis in our study had enough power to yield a credible result.

\section{Abbreviation}

ICU: Intensive care unit.

\section{Competing interests}

The authors declare that they have no competing interests.

Published: 05 Mar 2014

\section{References}

1. Ju M, Tu G, Han Y, He H, He Y, Mao H, Wu Z, Yin Y, Luo J, Zhu D, Luo Z, Xue Z: Effect of admission time on mortality in an intensive care unit in mainland China: a propensity score matching analysis. Crit Care 2013, 17:R230.

2. Andersen PK, Geskus RB, de Witte T, Putter H: Competing risks in epidemiology: possibilities and pitfalls. Int J Epidemiol 2012, 41:861-870.

3. Wolkewitz M, Vonberg R, Grundmann H, Beyersmann J, Gastmeier P, Baerwolff S, Geffers S, Behnke M, Rueden H, Schumacher M: Risk factors for the development of nosocomial pneumonia and mortality on intensive care units: application of competing risks models. Crit Care 2008, 12:R44.

4. Zwiener I, Blettner M, Hommel G: Survival analysis: part 15 of a series on evaluation of scientific publications. Dtsch Arztebl Int 2011, 108:163-169.

$10.1186 / \mathrm{cc} 13757$

Cite this article as: Wolkewitz and Sommer: Evaluating mortality in an intensive care unit requires extended survival models. Critical Care $2014,18: 415$ 